\title{
Measurement of the absolute reflectance of polytetrafluoroethylene (PTFE) immersed in liquid xenon
}

\author{
F. Neves,,$^{a, 1}$ A. Lindote, ${ }^{a}$ A. Morozov, ${ }^{a}$ V. Solovov, ${ }^{a}$ C. Silva, ${ }^{a}$ P. Bras, ${ }^{a}$ J. P. Rodrigues ${ }^{a}$ and \\ M. I. Lopes ${ }^{a}$ \\ ${ }^{a}$ LIP-Coimbra, Department of Physics, University of Coimbra, Rua Larga, 3004-516 Coimbra, Portugal \\ E-mail: neves@coimbra.lip.pt
}

\begin{abstract}
The performance of a detector using liquid xenon (LXe) as a scintillator is strongly dependent on the collection efficiency for xenon scintillation light, which in turn is critically dependent on the reflectance of the surfaces that surround the active volume. To improve the light collection in such detectors the active volume is usually surrounded by polytetrafluoroethylene (PTFE) reflector panels, used due to its very high reflectance - even at the short wavelength of scintillation light of LXe (peaked at $178 \mathrm{~nm}$ ). In this work, which contributed to the overall R\&D effort towards the LUXZEPLIN (LZ) experiment, we present experimental results for the absolute reflectance of three different PTFE samples (including the material used in the LUX detector) immersed in LXe for its scintillation light. The obtained results show that very high bi-hemispherical reflectance values $(\geq 97 \%)$ can be achieved, enabling very low energy thresholds in liquid xenon scintillator-based detectors.
\end{abstract}

KEYWORDS: noble liquid detectors, dark matter detectors, scintillation

\footnotetext{
${ }^{1}$ Corresponding author.
} 


\section{Contents}

1 Introduction 1

2 Method 3

3 Experimental setup $\quad 3$

4 Data analysis and results $\quad 5$

$\begin{array}{lll}4.1 & \text { Simulation } & 5\end{array}$

$\begin{array}{lll}4.2 & \text { Data analysis } & 7\end{array}$

$\begin{array}{llr}4.3 & \text { Results } & 9\end{array}$

5 Conclusions 12

\section{Introduction}

Liquid xenon (LXe) is widely used as sensitive medium in detectors for different applications ranging from astrophysics to medical imaging [1,2]. Most of these detectors are based on the collection of the xenon scintillation light. This is the case, for instance, of several detectors used in direct dark matter detection (e.g. ZEPLIN-II, LUX, XENON, PANDA-X) and other rare event search experiments (e.g. EXO, MEG).

The performance of these detectors (e.g. their energy resolution, discrimination capability and sensitivity) strongly depends on the amount of scintillation light collected, which, in turn, is critically dependent on the reflectance of the internal surfaces of the detector active region. Most common construction materials, in particular stainless steel (SS) and titanium, have very low reflectivity for the xenon scintillation light (peak $=178 \mathrm{~nm} ; \mathrm{FWHM}=14 \mathrm{~nm}[3]$ ). Hence, the detector active region is usually surrounded by a good reflector for light in this wavelength region.

Polytetrafluoroethylene (PTFE), a synthetic fluoropolymer, has proved to be the best choice of reflector for LXe scintillation-based detectors so far. Besides having a high Vacuum Ultra-Violet (VUV) reflectance (see below), it presents other very suitable properties: the manufacture process yields a highly radio-pure material (ppb in $\mathrm{U} / \mathrm{Th}$ ) $[4,5]$; it has good mechanical properties (despite a $1.4 \%$ thermal contraction at liquid xenon temperatures [6]); and outgassing rates are relatively low [7]. Unlike some metallic coatings, such as aluminum, which are also good VUV reflectors, its optical properties are stable against corrosion (e.g. from atmospheric $\mathrm{O}_{2}$ or $\mathrm{H}_{2} \mathrm{O}$ ) therefore not requiring any special handling or storing conditions [8]. Moreover, PTFE is chemically inert, has a high melting point and excellent dielectric properties [9].

The reflectance of PTFE has been extensively measured in gas and in vacuum for wavelengths in the range from $200 \mathrm{~nm}$ up to $3400 \mathrm{~nm}$ [10-12]. The results show a reflectance of about $99 \%$ for wavelengths between $350 \mathrm{~nm}$ and $1500 \mathrm{~nm}$. The reflectance decreases for ultraviolet light, being $93.4 \%$ at $200 \mathrm{~nm}$. It is also known that, at least between 250 and $3400 \mathrm{~nm}$, the optical properties differ for

low- and high-density PTFE [10]: in this wavelength range, low-density PTFE $(1.55 \mathrm{~g} / \mathrm{ml})$ shows a lower transmittance and a higher reflectance compared to the high-density material $(2.17 \mathrm{~g} / \mathrm{ml})$. 
Measurements between 120 and $220 \mathrm{~nm}$ using an Ulbricht sphere [13] show that the reflectance of an unspecified type of PTFE decreases slowly between 220 and $175 \mathrm{~nm}$, followed by a sharp drop at shorter wavelengths due the absorption edge of the PTFE $(161 \mathrm{~nm})$. At $178 \mathrm{~nm}$, the reflectance was measured to be about $56 \%$.

More recently, PTFE bi-hemispherical reflectance (BHR) and its angular distribution for gaseous xenon scintillation light (peaked at $175 \mathrm{~nm}$ ) were reported for several samples of PTFE manufactured by different processes (extruded, expanded, skived and pressed), along with those of other fluoropolymers, namely poly(ethene-co-tetrafluoroethene) (ETFE), hexafluoropropylene (FEP) and perfluoroalkoxy (PFA) [14]. All the samples were measured in an atmosphere of argon gas at room temperature. The measurements were carried out using a dedicated angle resolution system (goniometer), with the reflected light being sampled at a wide range of angles (including directions outside the plane of incidence). The obtained BHR for these PTFE samples ranges from about $47 \%$ to $70 \%$, depending on the manufacturing process and surface finishing. The best results were obtained with molded PTFE after polishing. The reflectance distribution of PTFE at $175 \mathrm{~nm}$ clearly shows specular and diffuse components, in agreement with what was previously reported for a single angle of incidence of $45^{\circ}$ at $172 \mathrm{~nm}[15]$.

When installed in LXe detectors, PTFE is immersed in liquid xenon and kept at low temperature $\left(\approx-100^{\circ} \mathrm{C}\right)$. Both these factors are expected to influence the optical properties of the PTFE surface, making it not trivial to extrapolate the expected response in these conditions based on the measurements performed in vacuum or gas [16]. Attempts of carrying out such an extrapolation have resulted in predictions which are systematically and significantly lower than the values $\mathcal{O}(>95 \%)$ estimated in LXe detectors using this material as a reflector (such as ZEPLIN-II, XENON100, and LUX) [16, 17]. PTFE reflectance in excess of $90-95 \%$ has also been reported for smaller LXe chambers $[18,19]$. In all these works, a lower limit for PTFE reflectance has been obtained by comparing the collected scintillation light produced by radioactive sources, internal or external, in a LXe chamber built for other purposes, with the expectation obtained by Monte-Carlo (MC) simulation of the light transport and collection in the chamber. Due to the dimensions and other features of those chambers, several other parameters apart from PTFE reflectance are also fitted (or their values fixed based on assumptions). Examples of those extra parameters are the quantum efficiency of the photomultipliers, the reflectivity of other materials existing in the active volume of the chamber (e.g. field grid wires) and the scintillation light attenuation and Rayleigh scattering lengths in liquid xenon. Moreover, those simulations have always assumed that reflections on the PTFE surfaces were purely diffuse, following the Lambert cosine law [20].

It is thus highly desirable to measure the absolute value of the reflectance of PTFE immersed in LXe for its scintillation light in a dedicated experiment and with a precision better than $O(1 \%)$ as a few percent difference in the PTFE reflectance may have a very significant impact on the threshold and sensitivity of the detector [8].

In this work, we report results of the absolute value of the reflectance of several samples of PTFE for xenon scintillation light and with PTFE directly immersed in LXe. To the best of our knowledge, these are the first measurements of this kind carried out in a dedicated experiment and using a method specifically designed for this purpose. This work was carried out in connection to the R\&D effort towards the LUX-ZEPLIN (LZ) experiment [8]. 


\section{Method}

Due to the constraint imposed by the need of having PTFE directly immersed in LXe, the high purity requirements for the detection of the xenon scintillation light and the low temperature $\left(\approx-100^{\circ} \mathrm{C}\right)$, it is not feasible to use one of the methods commonly employed in direct reflectance measurements (e.g. methods based on the use of a Ulbricht sphere or in an angle resolution scattering system). Also, one must keep in mind that a direct measurement of the reflectance using those methods is actually not possible and that detailed MC simulations of light propagation in the liquid are always necessary to account for Rayleigh scattering and light absorption in LXe, with the latter depending on the experimental conditions, namely, the cleanliness and outgassing from detector construction materials.

The method presented in this work is based on the measurement of the relative amount of light, produced directly in LXe by short-range mono-energetic alpha particles, that is collected at a photodetector after traversing a chamber where the geometry of the optical surfaces/volumes (PTFE/LXe) can be varied on the fly with high precision. This key feature allowed to measure light collection for different geometries, so varying the average number of reflections at the PTFE from just a couple to a few tens. Similarly, the average track length for optical photons in the liquid spans from a few centimeters to a couple of meters, depending on the liquid purity. The experimental results were then fitted with those from a detailed MC simulation of the light transport for each of the considered geometries, using the optical properties of PTFE and absorption length in the LXe as free parameters. Both the simulation and fitting procedures are explained in more detail in sec. 4 .

The chamber was built using only materials compliant with the purity requirements of LXe. In the active region, materials other than PTFE were kept to a bare minimum to reduce additional uncertainties from modeling their reflectances.

\section{Experimental setup}

The set-up used for the measurements reported here is schematically represented in fig. 1. Both the lateral and the top walls of the active region are made from $1 \mathrm{~cm}$ thick PTFE whose reflectance is intended to be measured. The lateral walls are arranged to form a cavity $150 \mathrm{~mm}$ long and with a square section of $10 \times 10 \mathrm{~mm}^{2}$. The top wall also has a square section of $10 \times 10 \mathrm{~mm}^{2}$ and can be moved by means of a micrometer drive along the length of the cavity, thus changing the chamber geometry through its height $h$ (see fig. 1). Centered on the top wall and recessed by $0.1 \mathrm{~mm}$ there is a ${ }^{241} \mathrm{Am}$ source $(6.8 \mathrm{~mm}$ in diameter) deposited on a stainless steel (SS) plate with a diameter of $8.46 \mathrm{~mm}$. The ${ }^{241} \mathrm{Am}$ source emits alpha particles with an energy of $5.486 \mathrm{MeV}$ at a rate of $\approx 1 \mathrm{kBq}$. Because these particles have a short range in LXe $(\approx 50 \mu \mathrm{m})$ compared with the dimensions of the active region of the chamber, we henceforth consider the energy of the alpha particle to be fully deposited in a point-like interaction. The energy deposited by each alpha particle in the LXe is then converted into $\approx 3.36 \times 10^{5}$ VUV photons from xenon scintillation (peak $\left.=178 \mathrm{~nm} ; F W H M=14 \mathrm{~nm}\right)[3,21]$. Some of those scintillation photons are absorbed by impurities in the LXe bulk volume or at any of the interfaces delimiting the active region (PTFE or SS from the source). The remaining photons, after traveling through the LXe volume and being reflected at the various surfaces, are detected by a photomultiplier (Hamamatsu R1668) placed at the bottom of the PTFE cavity and facing the ${ }^{241} \mathrm{Am}$ source. The PMT window has a diameter of $28.6 \mathrm{~mm}$ and is made of UV grade fused silica with a transmission of $\approx 90 \%$ for xenon scintillation light. There is a $1 \mathrm{~mm}$ gap between the PTFE walls and the PMT window to allow the displacement of LXe in and out from the cavity when moving the top wall and filling or emptying the chamber. 


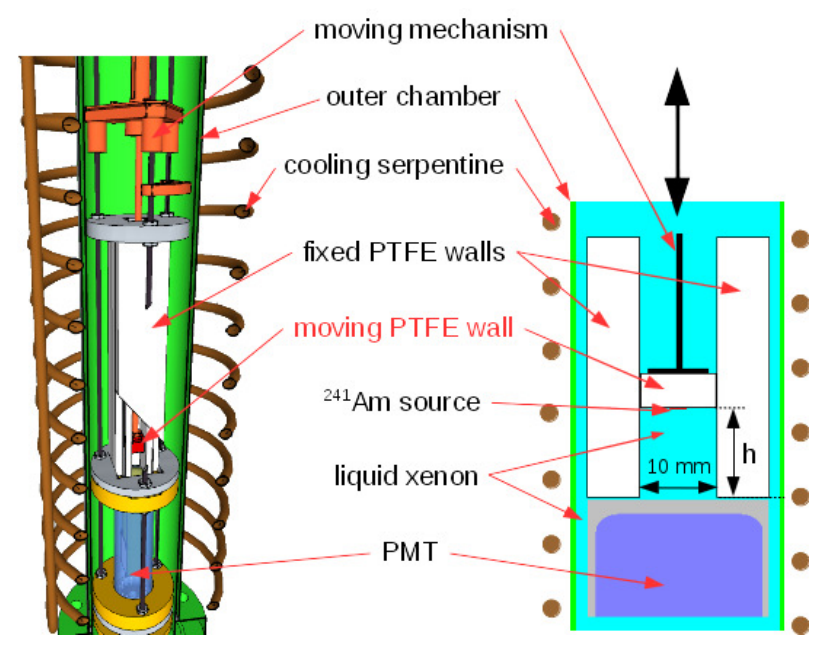

Figure 1. 3D view (left) and schematic representation (right) of the chamber showing the PTFE sample walls, the ${ }^{241} \mathrm{Am}$ source placed under the moving wall and the PMT at the bottom.

The described apparatus (fig. 1) is placed inside a vacuum tight cylindrical vessel, made of SS, connected to the xenon gas handling and purification system. All parts of the chamber, with the exception of the PMT, were cleaned in an ultrasonic bath of pure acetone prior to assembly. After closing, the chamber was heated up to $\approx 50^{\circ} \mathrm{C}$ and pumped until a vacuum of $<5 \times 10^{-8}$ mbar was achieved. This was followed by circulating xenon gas through the chamber and an Oxisorb purifier for at least 5 days. To control the purity of the xenon, the electron lifetime in the liquid phase was measured in a separate parallel plate chamber also connected to the gas system. The electron lifetime in the liquid is extracted directly from the pulse shape analysis of the charge signals due to a ${ }^{207} \mathrm{Bi}$ source placed at the cathode plate [22]. For all the reported measurements the electron lifetime was better than $40 \mu \mathrm{s}$ (at $1 \mathrm{kV} / \mathrm{cm})$, the highest value that can be measured with that chamber. This value allows to estimate the oxygen-equivalent concentration of electro-negative impurities in the LXe to be less than $17 \mathrm{ppb}$ [23]. Although being used as a control parameter of the LXe purity across the various measurements, in fact this value cannot be used to calculate the absorption length for the xenon scintillation light as will be discussed in sec. 4.3.

The setup was cooled down to $-103 \pm 1^{\circ} \mathrm{C}$ by placing the chamber in a cooled ethylene bath. The temperature of this bath is controlled by the amount of liquid nitrogen circulating through a copper coil surrounding the outer vessel (fig. 1) and an heater placed at the bottom of the bath. The chamber is filled with $\sim 1.5$ bar of xenon and left to thermalize for $\sim 12$ hours prior to start condensing xenon. Xenon is then slowly condensed into the chamber until it completely covers the PTFE walls. During condensation the position of the liquid level is monitored using a capacitive level sensor placed inside the outer chamber but outside the PTFE walls. The level of the liquid along with its temperature and the pressure in the gas phase are continuously monitored and stored for future reference.

For each position of the top wall $(h)$ the light spectrum is acquired and the position of the peak, $I(h)$, corresponding to the full energy deposition from the alpha particles in the liquid is estimated from a Gaussian fit (fig. 2). For each PTFE sample the values of $I$ were obtained for $h$ between 19 and $145 \mathrm{~mm}$ in steps of $7 \mathrm{~mm}$, both while moving down the top wall closer to the PMT window and then back up to the farthest position. This scan was repeated to look for any systematic effects, for example, from liquid purity degradation with time or bubble formation at the lateral PTFE walls. To 

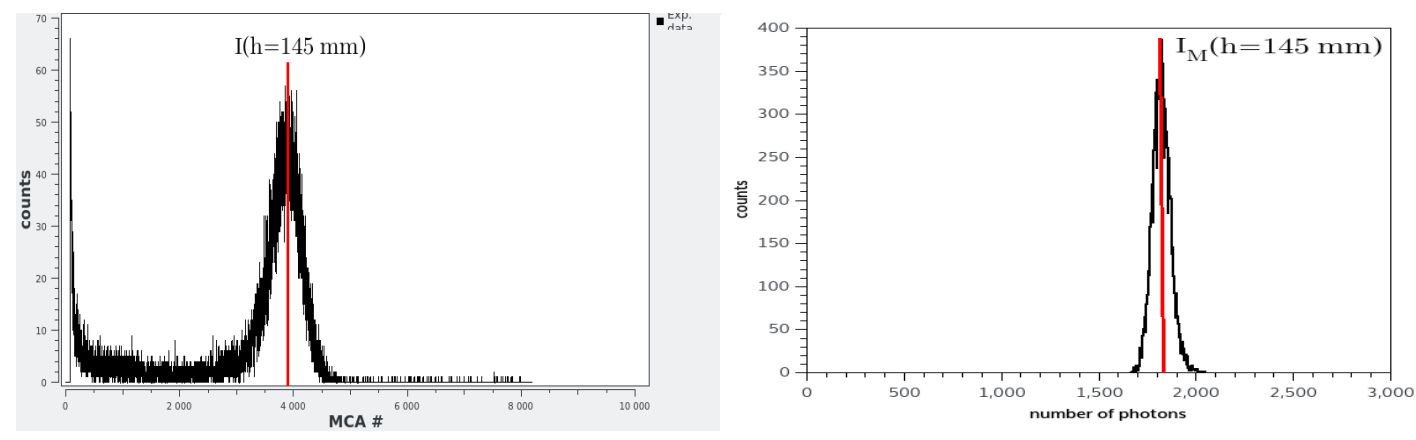

Figure 2. Example of an experimental spectrum obtained using one of the PTFE samples with the source at $h=145 \mathrm{~mm}$ (left) ; Example of a simulated spectrum of the number of photons crossing the PMT window also for $h=145 \mathrm{~mm}$ (right). The values of $I(145)$ and $I_{\mathcal{M}}(145)$ (where $\mathcal{M}$ tags the optical model used to simulate the PTFE reflectance - sec. 4.2) are also represented and were obtained by fitting a Gaussian to the correspondent peak in the spectrum.

test for reproducibility, the above scanning procedure was also repeated during successive cooldown cycles separated by a complete warm up of the chamber. It was observed that for each PTFE sample the values of $I(h)$ from multiple scans fully agree within the statistical errors and were therefore merged into a single dataset for subsequent analysis.

It was observed that the variation of the temperature of the liquid xenon induced a relative variation in the response of the PMT of $(0.013 \pm 0.001)^{\circ} \mathrm{C}^{-1}$. This is attributed to the fact that both the PMT and its voltage divider are immersed directly in the liquid. All data points presented in this work were therefore corrected for temperature variations and normalized to a liquid temperature of $-103^{\circ} \mathrm{C}$.

The PMT anode signals are pre-amplified using a Canberra 2005 module placed outside the chamber (at room temperature) and then fed into a Canberra 2020 amplifier for shaping and further amplification. The shaped signals are digitalized using a multi-channel analyzer (PTG 8008) and the corresponding spectra stored for offline analysis.

\section{Data analysis and results}

\subsection{Simulation}

As outlined in sec. 2, to determine the reflectance from $I(h)$ (sec. 3) the variation of the light collection with the chamber geometry $h$ has to be modeled by MC simulation. For that, a detailed simulation of the transport of the scintillation photons for each of the considered geometries was implemented using the ANTS2 software toolkit [24]. Only the inner chamber was simulated, as the details of the outer cylindrical vessel, structural support and instrumentation elements are irrelevant for light propagation inside the inner cell. The results from the ANTS2 simulations were validated at an early stage against those obtained using the GEANT4 toolkit [25] for the exact same conditions. As the results obtained using both packages agree within statistical errors, ANTS2 was selected for the rest of this study due to the considerably shorter computational time required for the simulation of the propagation of such a large number of initial scintillation photons.

For the simulation of the light transport in the inner cell, the relevant physical processes to be modeled are the propagation of the optical photons in the bulk LXe volume and their behavior on 
the interfaces. For the propagation of light in xenon, Rayleigh scattering and bulk absorption in LXe were considered: the interaction length for the former was set to $29 \mathrm{~cm} \mathrm{[26],} \mathrm{while} \mathrm{the} \mathrm{latter} \mathrm{(which} \mathrm{is}$ strongly affected by xenon purity) was left as a free parameter and allowed to vary during the analysis stage. Given that most of the inner cavity is surrounded by the PTFE for which we intend to measure the reflectance, the large majority of the interactions of the optical photons are with this material. For the simulation of the behavior of photons in the xenon/PTFE interfaces two models were considered. The first model $(D)$ considers only diffuse reflection following the Lambert cosine law

$$
d R_{d i f}\left(\theta_{r}\right)=\operatorname{Acos}\left(\theta_{r}\right) d \theta_{r},
$$

where $R_{d i f}\left(\theta_{r}\right)$ represents the probability of the light being reflected back into the LXe with an angle $\theta_{r}$ relative to the PTFE surface normal. The free parameter $A$ is the albedo, a constant representing the integral probability of the light not being absorbed at the PTFE. The second model $(D S)$ considers specular reflection at the liquid/PTFE interface and diffuse reflection of the photons that are refracted into the PTFE. To calculate the probability of specular reflection at any given angle of incidence $\left(\theta_{i}\right)$ the Fresnel equations were considered for non-polarized light:

$$
R_{\text {spec }}\left(\theta_{i}\right)=\frac{1}{2}\left(R_{p}\left(\theta_{i}\right)+R_{s}\left(\theta_{i}\right)\right)
$$

where $R_{p}\left(\theta_{i}\right)$ and $R_{s}\left(\theta_{i}\right)$ represent, respectively, the probability of reflection for p- and s-polarized light [20]:

$$
R_{p}\left(\theta_{i}\right)=\left(\frac{n^{2} \cos \theta_{i}-\sqrt{n^{2}-\sin ^{2} \theta_{i}}}{n^{2} \cos \theta_{i}+\sqrt{n^{2}-\sin ^{2} \theta_{i}}}\right)^{2}
$$

and

$$
R_{s}\left(\theta_{i}\right)=\left(\frac{\cos \theta_{i}-\sqrt{n^{2}-\sin ^{2} \theta_{i}}}{\cos \theta_{i}+\sqrt{n^{2}-\sin ^{2} \theta_{i}}}\right)^{2},
$$

with $n=n_{P T F E} / n_{L X e}$, where $n_{P T F E}$ and $n_{L X e}$ correspond to the refractive index of PTFE and liquid xenon, respectively. The value of $n_{L X e}$ was considered to be constant and set to 1.69 [27] while $n_{P T F E}$ was left as a free parameter. The probability of diffuse reflection can then be expressed as

$$
R_{\text {dif }}\left(\theta_{i}\right)=A\left[1-R_{\text {spec }}\left(\theta_{i}\right)\right]
$$

where $1-R_{\text {spec }}\left(\theta_{i}\right)$ is the probability of light being refracted into the PTFE bulk volume. The direction of this light when exiting the PTFE also follows the Lambert cosine law as for the $D$ model (eq. 4.1)

$$
d R_{d i f}\left(\theta_{r} \mid \theta_{i}\right)=R_{d i f}\left(\theta_{i}\right) \cos \left(\theta_{r}\right) d \theta_{r} .
$$

It is worth noting that the $D S$ model coincides with the $D$ model when $n_{P T F E}=n_{L X e}$ corresponding to $R_{\text {spect }}=0$. In this particular case eq. 4.5 can be written as $R_{d i f}=A$, having no dependence on $\theta_{i}$, $n_{P T F E}$ or $n_{L X e}$.

Apart from PTFE, the only materials where light can be reflected inside the inner cell are the SS in the ${ }^{241} \mathrm{Am}$ source and the fused silica in the PMT window. Despite of contributing only with a very small fraction to the total internal area of the chamber, the SS surface is the first interface for $\approx 50 \%$ of the photons, that are produced just above the source along the very short range $(\approx 50 \mu \mathrm{m})$ alpha particle tracks. As the composition of this SS is unknown, we tested two pairs of refractive 

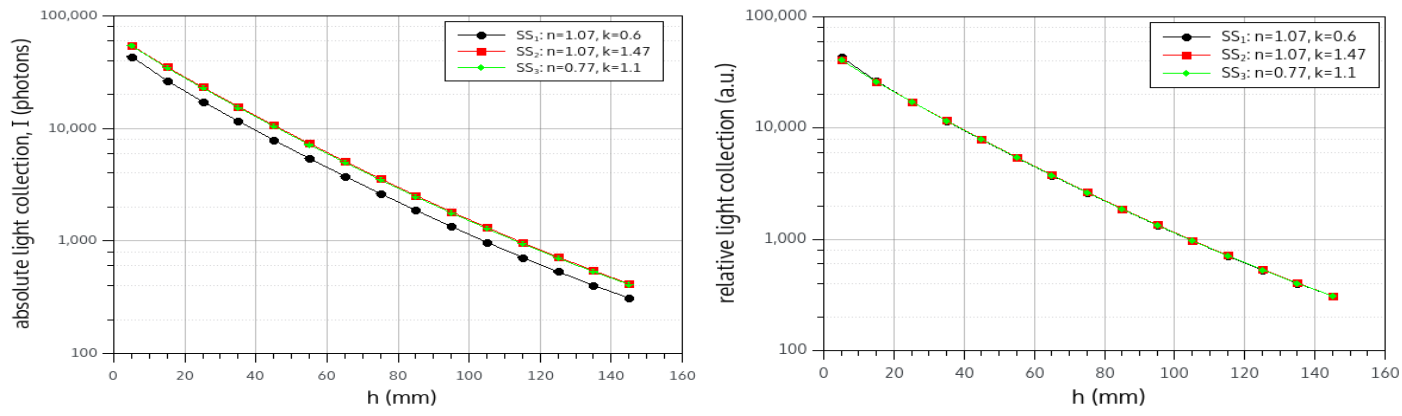

Figure 3. Example of the simulated absolute light collection as a function of the chamber height $(h)$ for $A=0.95, n_{P T F E}=1.75$ (DS model) and three different SS refractive indexes of the ${ }^{241}$ Am source (left). Despite the significant different refractive indexes and correspondent values of the absolute light collection, the scaled light collection profiles match perfectly within the statistical errors (right).

index values $((n, k)=(0.77,1.1)$ and $(n, k)=(1.07,1.47))$ found in the literature [28] (corresponding to two samples of SS with different compositions), and an additional third value $((n, k)=(1.07,0.6))$ which was estimated using data available from a previous experiment performed by our group for yet a different SS [27]. Despite the large differences in total reflectivity between the tested refractive indexes $(10 \%-30 \%$ for normal incident light), leading to a very different number of detected photons for every $h$ (fig. 1), it was verified that the scaled light collection profiles match perfectly within statistical errors (fig. 3). The best match between any two of the light collection profiles (for different SS refractive indexes) was obtained by multiplying one of them by a scaling factor $C$ and minimizing the difference between the scaled and unscaled profiles using $C$ as a free parameter. As not affecting the relative light collection (see sec. 4.2), the result of all simulations presented in this work were done considering a refractive index for the SS of $n=1.07$, with a complex factor $k=0.6$ accounting for the attenuation at the SS.

Regarding the PMT, to our best knowledge, its quantum efficiency (QE) at LXe temperature is somewhere in the range of $15 \%-30 \%$ for the xenon scintillation light. As for the case of the SS reflectivity, different values of $\mathrm{QE}$ result in a simple scaling in the number of detected photons as a function of $h$. For that reason all the photons arriving at the PMT window and refracted inside are considered to be detected with $100 \%$ efficiency, thus also disregarding any loss due to the window thickness as well as any inefficiency in the collection of the the photoelectrons on the first dynode. The light reflection at the PMT window was considered to be purely specular with an index of refraction of the fused silica equal to 1.59 [29].

\subsection{Data analysis}

The PTFE reflectance is obtained by fitting the measured relative light collection, $I(h)$, with the prediction of the Monte Carlo simulation. As detailed in the previous section, the uncertainties in both the reflectivity of the SS and QE of the PMT do not allow for a direct comparison of the experimental and simulated results in terms of the absolute number of photons arriving at the PMT photocathode. Instead, we compare the relative variation of the light collected with $h$ assuming that both the SS reflectivity and PMT QE contributions correspond to a scaling factor. 
For the simplest model considering only diffuse reflection in the PTFE $(D)$, the free fitting parameters were the PTFE albedo ( $A$, eq. 4.1) and the attenuation length $(\lambda)$ for the scintillation light in LXe. When considering specular reflection $(D S)$, the refractive index of the PTFE $\left(n_{P T F E}\right.$, eq. 4.2$)$ is an additional free parameter. For each source position $(h)$ and set of parameters $\mathcal{O}_{D}=\{A, \lambda\}$ or $\mathcal{O}_{D S}=\left\{A, n_{P T F E}, \lambda\right\}$, depending on the model, we sample the isotropic emission of $10^{5}$ scintillation photons per alpha particle for a total of 300 alpha particles distributed uniformly over the ${ }^{241} \mathrm{Am}$ source surface. The simulated number of scintillation photons per alpha particle is $\approx 1 / 3$ of the real number of photons one expects to be produced in the liquid (sec. 3). Since we are only interested in the relative variation of the light collection and to save computational resources, we reduced the number of emitted photons in the simulation while keeping the statistical errors on $I_{\mathcal{M}}$ lower than $\mathcal{O}(0.1 \%)\left(I_{\mathcal{M}}(h) \gtrsim 1000\right)$, where $\mathcal{M}$ stands for the $D$ or $D S$ model. Given the CPU time required per simulation, we chose to generate a multidimensional template grid for each of the reflectivity models. This approach also allows for a more systematic search of local minima and possible degeneracy of solutions. For any given set of parameters $\mathcal{O}_{\mathcal{M}}$, the simulated response $I_{\mathcal{M}}\left(\mathcal{O}_{\mathcal{M}} ; h\right)$ is obtained using a bilinear interpolation at the correspondent grid nodes. Henceforth and for the sake of simplicity, the subscript $\mathcal{M}$ will be omitted from the set of optical parameters $\mathcal{O}_{\mathcal{M}}$ when the model is already defined by the calling function (e.g. $I_{\mathcal{M}}\left(\mathcal{O}_{\mathcal{M}} ; h\right) \equiv I_{\mathcal{M}}(\mathcal{O} ; h)$ ).

For the diffuse only model $(D)$ a $39 \times 50 \times 29$ grid was generated (56550 individual simulations) within the corresponding ranges: $A=[0.6,1], \lambda=[100,5000] \mathrm{mm}$ and $h=[5,145] \mathrm{mm}$. Both the $\lambda$ and $h$ axis are regular with a step size of $100 \mathrm{~mm}$ and $5 \mathrm{~mm}$, respectively. For the $A$ axis, we refined the step size from 0.05 for $A \leq 0.7$ to 0.01 in the range $0.7<A<0.9$ and 0.005 for $A \geq 0.9$, corresponding to the region of interest for all the samples measured in this work. For the model considering specular reflection $(D S)$ a $16 \times 29 \times 29 \times 29$ grid was generated (corresponding to a total of 390224 simulations) with the same ranges defined above for the $A, \lambda$ and $h$ axis. The refractive index $\left(n_{P T F E}\right)$ is varied in the range $[1.3,1.9]$ with a step size of 0.01 for $1.65 \leq n_{P T F E} \leq 1.9$ and 0.05 below 1.65 . Given the quite large number of simulations and corresponding $\mathrm{CPU}$ time required to populate the 4-dimensional grid for the $D S$ model, the generated grid is sparser than for the former case in all parameters but $h$ : the step size for the $\lambda$ axis was kept at $100 \mathrm{~mm}$ only for $\lambda<1200 \mathrm{~mm}$, where the variation of the light collected is steeper, and increased to $200 \mathrm{~mm}$ for higher attenuation lengths. Similarly, the albedo axis step size was increased by a factor of 2 when compared with the $D$ model, being 0.01 in the region of interest, i.e. $A \geqslant 0.9$. As a general rule to refine the grid, the interpolation errors were kept within the level of the statistical errors coming from the simulation on the number of photons arriving at the PMT window.

For a given set of experimental points corresponding to a PTFE sample, the relative variation of the light collected with $h$ is compared with the simulated response $\left(I_{\mathcal{M}}\right)$ after scaling the latter by a constant value $C$. Explicitly, the value of $C$ is obtained by minimizing

$$
\chi_{\mathcal{M}}^{2}(C ; \mathcal{O})=\frac{1}{N-N_{\mathcal{O}}-1} \sum_{i} \frac{\left[I\left(h_{i}\right)-C \times I_{\mathcal{M}}\left(\mathcal{O} ; h_{i}\right)\right]^{2}}{\sigma_{e x p}^{2}\left(h_{i}\right)+\sigma_{\mathcal{M}}^{2}\left(\mathcal{O} ; h_{i}\right)+\mathcal{H}_{\mathcal{M}}^{2}\left(\mathcal{O} ; h_{i}\right)}
$$

where $i$ represents a data point measured at a given source position $h_{i}, N$ is the total number of experimental points in the data set and $N_{\mathcal{O}}$ the number of free parameters for the model being fitted $\left(N_{D}=2, N_{D S}=3\right)$. The best set of optical parameters is then found by simply iterating through the grid space and finding the set of parameters $\mathcal{O}_{\mathcal{M}}$ yielding the smaller value for $\chi_{\mathcal{M}}(\mathcal{O})$ (eq. 4.7). The size of the iteration steps is defined over the region of interest based on the variation of $\chi_{\mathcal{M}}$ with $\mathcal{O}_{\mathcal{M}}$, and set typically to be a factor of $\lesssim 0.1$ smaller than the 1 sigma region in $\chi_{\mathcal{M}}$ around 
the absolute minimum. The statistical experimental errors, $\sigma_{\text {exp }}\left(h_{i}\right)$ are quadratically added to the statistical errors from the simulation, $\sigma_{\mathcal{M}}\left(\mathcal{O} ; h_{i}\right)$, and to $\mathcal{H}_{\mathcal{M}}\left(\mathcal{O} ; h_{i}\right)$, which takes into account the uncertainties in the determination of the source position. In order to propagate the uncertainties in $h\left(\sigma_{h}\right)$, we consider the quadratic variation of $I_{\mathcal{M}}$ at the limits of the interval $\left[h-\sigma_{h}, h+\sigma_{h}\right]$ to be representative of the uncertainties in the light collection from the determination of $h$, i.e.:

$$
\mathcal{H}_{\mathcal{M}}^{2}(\mathcal{O} ; h)=\left(I_{\mathcal{M}}\left(\mathcal{O} ; h-\sigma_{h}\right)-I_{\mathcal{M}}(\mathcal{O} ; h)\right)^{2}+\left(I_{\mathcal{M}}\left(\mathcal{O} ; h+\sigma_{h}\right)-I_{\mathcal{M}}(\mathcal{O} ; h)\right)^{2}
$$

It was verified, with the moving mechanism exposed inside a clean room, that the uncertainty from repositioning the source at any given position between $h=0 \mathrm{~mm}$ and $h=150 \mathrm{~mm}$ was $\sigma_{h} \lesssim 0.1 \mathrm{~mm}$ when measuring its position relatively to the two stoppers placed at both ends of the allowed course of movement.

\subsection{Results}

The reflectance of three samples of PTFE was measured: $807 N X$ and NXT85 from Applied Plastics Technology (APT) and 8764 from Technetics. To the best of our knowledge there is no published data concerning the reflectance of the first two sample materials for the xenon scintillation light at $-100^{\circ} \mathrm{C}$ and when immersed in LXe. The 3rd sample corresponds to the PTFE used in the LUX experiment to define the active region, thus allowing a direct comparison with the reflectance estimated $\mathrm{b}$ the LUX collaboration $[17,30]$.

Figure. 4, shows the fitting results for the three measured PTFE samples using the diffuse only $(D)$ model. It can be observed that this model systemically underestimates the light detected for $h \lesssim 40 \mathrm{~mm}$, which is attributed to the absence of specular reflection in the $D$ model. In fact, for small values of $h$ the average number of reflections decreases and thus a single specular reflection can provide a direct path to the PMT window. On the other hand, as the average number of reflections increases with $h$, the diffuse reflection (which is characterized by large values of $A$ for the samples studied) becomes dominant thus suppressing the weight of possible forward specular-only trajectories.

The above interpretation is strengthened when fitting the experimental results with the model which takes into consideration the specular reflection at the PTFE walls. As can be seen in fig. 5, the $D S$ model succeeds to reproduce the measured relative light collection for all three PTFE samples.

The results obtained for all the PTFE samples using both reflectivity models considered in this work are summarized in tab. 1 . It is worth noting that, as it can be seen in figs. 4 and 5 , the absolute values of the light collected, $I(h)$ (expressed in a.u.), are scaled accordingly to the corresponding PTFE reflectivities (tab. 1) for all heights and especially for higher values of $h$, where the larger number of reflections strongly enhance the effect of small differences in the reflectance.

Figure 6 shows a cut of the $95 \%$ confidence level region from fitting the NXT85 experimental data using the $D S$ model and illustrates the existence of a degeneracy between the values of $\lambda$ and $A$, quantities characterizing the extinction of photons in the liquid and the PTFE, respectively. The profile of this degeneracy is common to all PTFE samples and just exposes the inability to resolve which process is actually responsible for the absorption of the photons. Although the lifetime of free electrons (sec. 3) is a proxy for the concentration of electronegative impurities in the liquid, there is no direct way to use this information to independently estimate the actual attenuation length $(\lambda)$ since we do not know which species are actually diluted in the LXe.

Given the impossibility to further constrain the attenuation length in the liquid, the uncertainties on the fitting parameters defining the reflectance of the PTFE ( $A$ and $n_{P T F E}$ ) were quantified by the boundaries of their $95 \%$ confidence regions over the entire range of values of $\lambda$ considered in this 


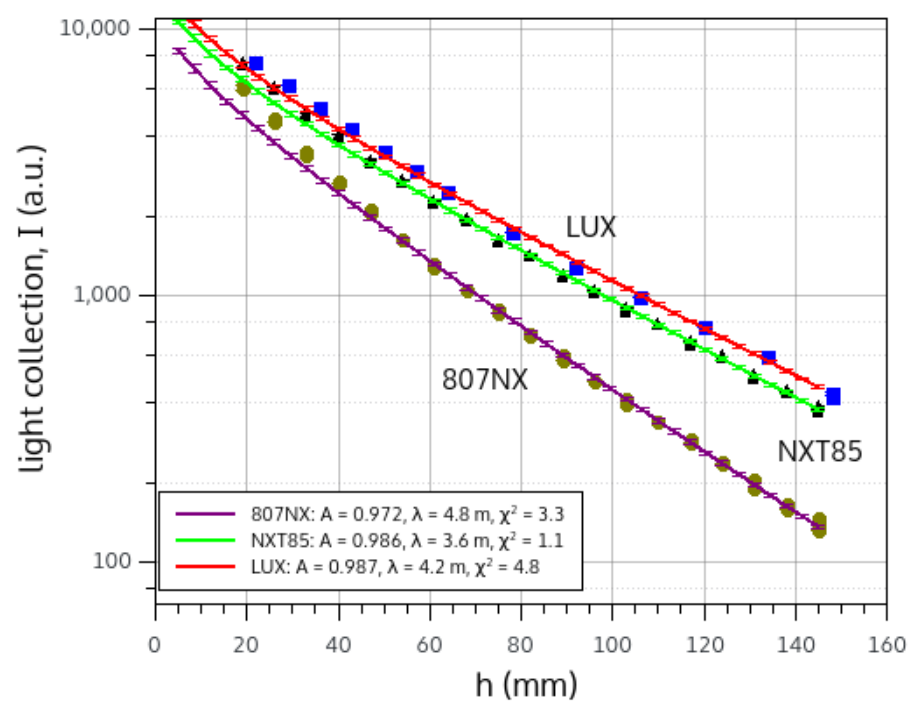

Figure 4. Fit (purple, green and red lines) to the experimental data (dark yellow circles, black triangles and blue squares) using the diffuse-only model $(D)$ for the three samples of PTFE measured, respectively: 807NX, NXT85 and 8764 (LUX). The best fitting parameters and corresponding $\chi_{D}^{2}$ (eq. 4.7) are also indicated for each sample.

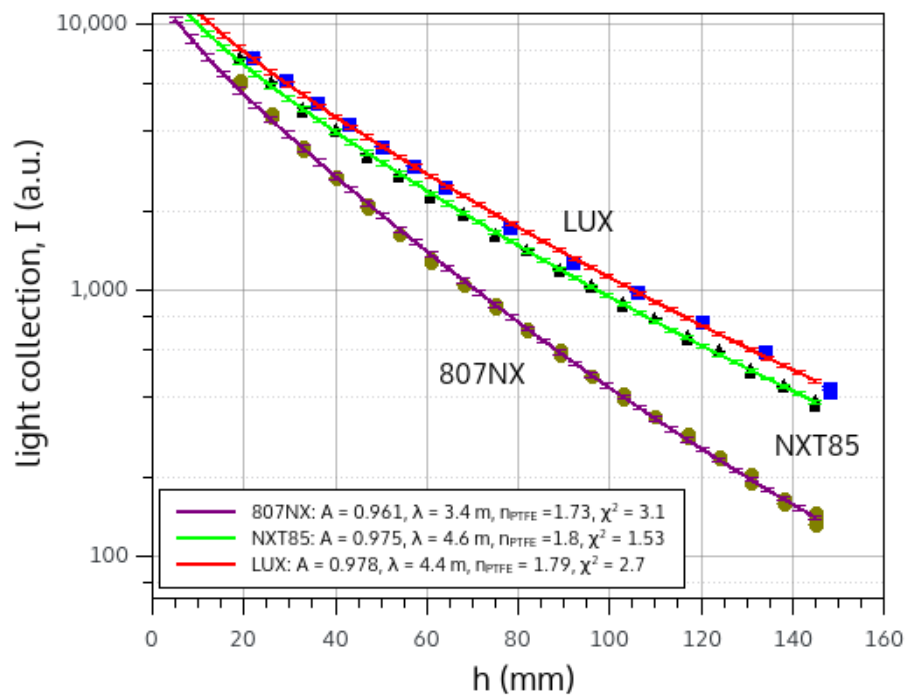

Figure 5. Fit (purple, green and red lines) to the experimental data (dark yellow circles, black triangles and blue squares) using the diffuse plus specular $(D S)$ reflection model for the three samples of PTFE measured, respectively: $807 \mathrm{NX}$, NXT85 and 8764 (LUX). The best fitting parameters and corresponding $\chi_{D S}^{2}$ (eq. 4.7) are also indicated for each sample. 


\begin{tabular}{|c|c|c|c|c|c|c|}
\hline & \multicolumn{2}{|c|}{ Diffuse model $(D)$} & \multicolumn{4}{|c|}{ Diffuse + Specular model $(D S)$} \\
\hline & $A$ & $\lambda(\mathrm{mm})$ & $A$ & $n_{P T F E}$ & $\lambda(\mathrm{mm})$ & $B H R$ \\
\hline $807 \mathrm{NX}$ & $\begin{array}{c}0.972 \\
(>0.97)\end{array}$ & 4800 & $\begin{array}{c}0.961 \\
(>0.955)\end{array}$ & 1.73 & 4600 & $\begin{array}{c}0.961 \\
(>0.955)\end{array}$ \\
\hline NXT85 & $\begin{array}{c}0.986 \\
(>0.984)\end{array}$ & 3600 & $\begin{array}{c}0.975 \\
(>0.973)\end{array}$ & 1.8 & 4600 & $\begin{array}{c}0.975 \\
(>0.973)\end{array}$ \\
\hline LUX & $\begin{array}{c}0.987 \\
(>0.985)\end{array}$ & 4200 & $\begin{array}{c}0.978 \\
(>0.975)\end{array}$ & 1.79 & 3000 & $\begin{array}{c}0.978 \\
(>0.975)\end{array}$ \\
\hline
\end{tabular}

Table 1. Best fitting parameters to the experimental data for the three PTFE samples measured in this work using both the $D$ and $D S$ model for the PTFE reflectance. For the albedo $(A)$ the lower $95 \%$ confidence boundary is also shown. For the $D S$ model the values of the Bi-Hemispherical Reflectance (BHR) are represented for the best fit parameters and at the lowest $95 \%$ confidence boundary. Note that for the $D$ case the $B H R$ equals $A$ by definition of the model. For the $D S$ model, the presented values of the BHR also equals $A$ for all the studied PTFE samples and within the precision of the measurement. However, in this case this is just a consequence of the relative importance of both components of reflection (diffuse/specular) in the BHR computation which gives little weight to grazing angles where the specular reflection is more important.

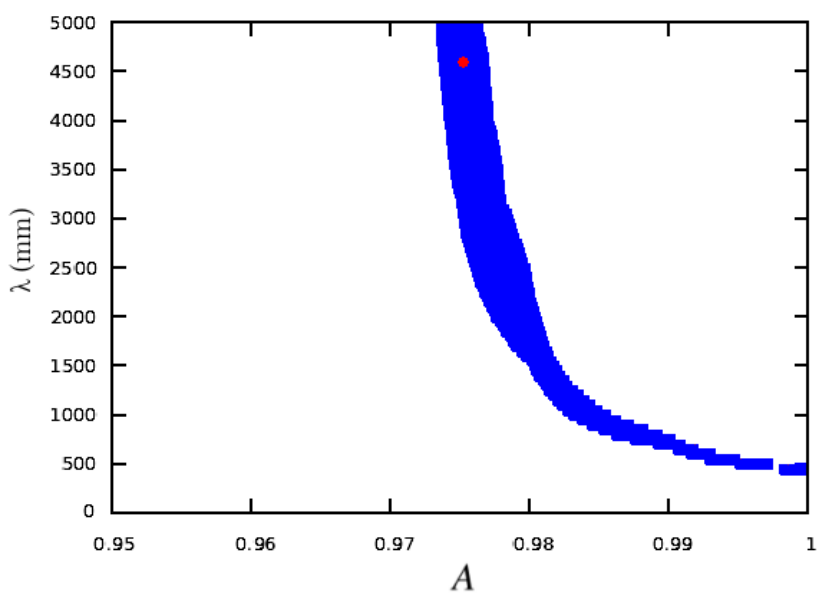

Figure 6. Best fit using the $D S$ model of the NXT85 experimental data (red dot); and, cut $\left(n_{P T F E}=1.8\right)$ across the $\lambda$ and $A$ parameters space of the corresponding $95 \%$ confidence region (blue band).

work. The upper and lower boundaries of these regions are represented in fig. 7 for the albedo $(A)$ and refractive index of the PTFE $\left(n_{P T F E}\right)$ for the $D$ and $D S$ models. The lowest values of the lower $95 \%$ confidence boundary for the albedo $\left(A_{\min }\right)$ are also summarized in tab. 1. For all measured PTFEs samples, $A_{\min }$ values correspond to $\lambda=5 \mathrm{~m}$, as the boundaries asymptote for higher values of the attenuation length. The values of the Bi-Hemispherical Reflectance (BHR) for the $D S$ model corresponding to both the best fit parameters and the 95\% CL lower limits are also shown in tab. 1 (the latter in parenthesis). The BHR values are obtained for a white sky illumination [31] and are dominated by the correspondent value of $A$, being almost insensitive to $n_{P T F E}$ within its $95 \%$ confidence region at any attenuation length. The reason for this is that the incident grazing angles for which $R_{\text {spec }}$ 

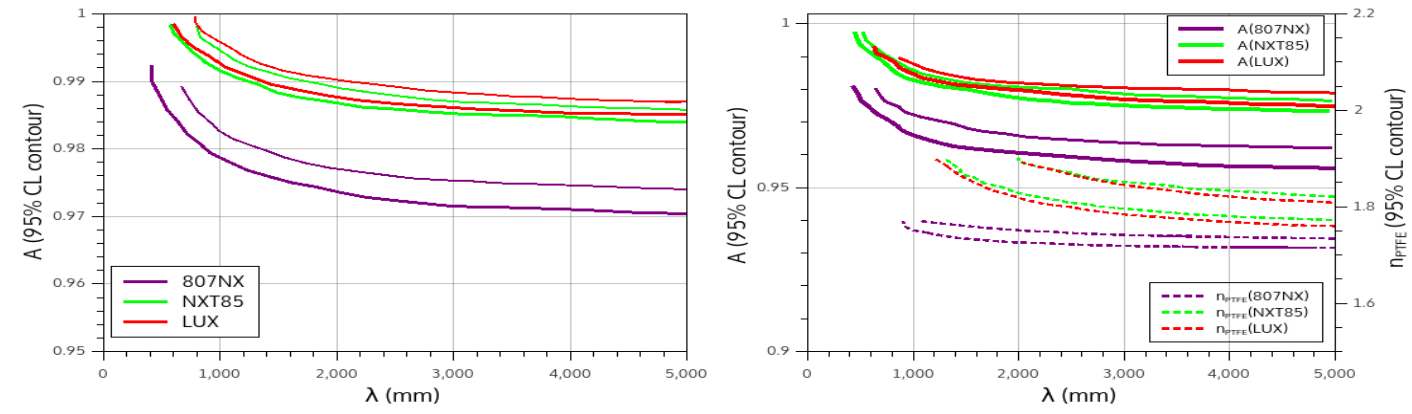

Figure 7. Upper and lower boundaries of the $95 \%$ confidence regions for the fitting parameters $A$ (solid lines) and $n_{P T F E}$ (broken lines) as a function of $\lambda$ for both the $D$ (left) and $D S$ (right) models.

(eq. 4.2) has a higher contribution are strongly suppressed under a white sky distribution. Also note that by definition of the $D$ model, the BHR simply equals the corresponding value of $A$.

The BHR values obtained using the parameters $\mathcal{O}_{D S}=\left\{A_{\text {min }}, n_{P T F E}, \lambda=5 \mathrm{~m}\right\}$ and $\mathcal{O}_{D}=$ $\left\{A_{\text {min }}, \lambda=5 \mathrm{~m}\right\}$, shown in table 1 , represent for each PTFE sample the lowest reflectance that can be taken from this work at a $95 \%$ confidence level. The relevance of these values relates directly to the performance of LXe detectors using PTFE reflectors as they define a lower limit for the detection threshold in terms of light collection.

It is worth mentioning that relative reflectance measurements as a function of the PTFE thickness were also carried out in a different setup for samples of both the 807NX and NXT85 materials immersed in LXe [32]. The obtained results for the scintillation light of LXe show no appreciable reduction in the reflectance from $9.5 \mathrm{~mm}$ down to $1 \mathrm{~mm}$ wall thickness. They also provide the indication that the reflectance of NXT85 is slightly larger than that of 807NX, in agreement with the results obtained in this work for the absolute reflectance of those PTFE for the xenon scintillation light.

\section{Conclusions}

The absolute reflectance of three PTFE samples immersed in LXe was measured for the xenon scintillation light $(\lambda=178 \mathrm{~nm})$. To the best of our knowledge, these are the first measurements of the PTFE reflectance immersed in LXe to be performed using a dedicated setup optimized for that purpose.

The experimental method used proved to be sensitive to small differences, $\mathcal{O}(0.1 \%)$, in the absolute reflectance of the PTFE samples. The obtained results confirm the higher reflectance of PTFE at $\lambda=178 \mathrm{~nm}$ when immersed in LXe when compared with extrapolations from existing measurements performed in gas and at room temperature. This is in agreement with previous observations in large and medium size detectors built for other purposes. Furthermore, the results show that very high reflectances $(>97 \%)$ can be attained for xenon scintillation light when using PTFE immersed in LXe. The results also support the existence of a specular reflection component, which is usually not considered in the simulation of LXe detectors based on xenon scintillation and using PTFE as a light reflector. The addition of this specular component may be very important (depending on the geometry) for the correct reconstruction of events near the PTFE walls in such detectors (e.g. LZ, LUX, XENON). The ability to choose and characterize the PTFE reflectance early in the design stage may play a crucial role in the optimization of future LXe detectors, especially in what concerns their detection threshold and sensitivity. 


\section{Acknowledgments}

We would like to acknowledge the LZ and LUX collaborations for providing the PTFE materials and

for many useful discussions. This work was supported by funding from Fundação para a Ciência e a Tecnologia (FCT) through the Project-Grant No. PTDC/FIS-NUC/1525/2014.

\section{References}

[1] E. Aprile and T. Doke, "Liquid xenon detectors for particle physics and astrophysics," Rev. Mod. Phys., vol. 82, pp. 2053-2097, Jul 2010. [Online]. Available:

http://link.aps.org/doi/10.1103/RevModPhys.82.2053

[2] V. Chepel and H. Araújo, "Liquid noble gas detectors for low energy particle physics," Journal of Instrumentation, vol. 8, no. 04, p. R04001, 2013. [Online]. Available: http://stacks.iop.org/1748-0221/8/i=04/a=R04001

[3] J. Jortner, L. Meyer, S. A. Rice, and E. G. Wilson, "Localized excitations in condensed Ne, Ar, Kr, and Xe," The Journal of Chemical Physics, vol. 42, no. 12, pp. 4250-4253, 1965. [Online]. Available: http://scitation.aip.org/content/aip/journal/jcp/42/12/10.1063/1.1695927

[4] D. Akerib et al., "Radiogenic and muon-induced backgrounds in the LUX dark matter detector," Astroparticle Physics, vol. 62, pp. 33 - 46, 2015. [Online]. Available: http://www.sciencedirect.com/science/article/pii/S0927650514001054

[5] E. Aprile et al., "Material screening and selection for XENON100," Astroparticle Physics, vol. 35, no. 2, pp. 43 - 49, 2011. [Online]. Available:

http://www.sciencedirect.com/science/article/pii/S0927650511000971

[6] R. K. Kirby, "Thermal expansion of polytetrafluoroethylene (teflon) from $-190^{\circ}$ to $+300^{\circ}$ C," Journal of Research of the National Bureau of Standards, vol. 57, no. 2, pp. 91-94, 1956.

[7] K. Poole and M. Michaelis, "Hialvac and Teflon outgassing under ultra-high vacuum conditions," Vacuum, vol. 30, no. 10, pp. 415 - 417, 1980. [Online]. Available: http://www.sciencedirect.com/science/article/pii/S0042207X80800558

[8] D. Akerib et al., "LUX-ZEPLIN (LZ) conceptual design report," 2015. [Online]. Available: arXiv:1509.02910

[9] M. Chant, "Dielectric properties of some insulating materials over the temperature range 4.2-300 k," Cryogenics, vol. 7, no. 6, pp. 351 - 354, 1967. [Online]. Available: http://www.sciencedirect.com/science/article/pii/0011227567900094

[10] B. K. Tsai, D. W. Allen, L. M. Hanssen, B. Wilthan, and J. Zeng, "A comparison of optical properties between solid PTFE (Teflon) and (low density) sintered PTFE," Proc. SPIE, vol. 7065, pp. 70 650Y-70 650Y-9, 2008. [Online]. Available: http://dx.doi.org/10.1117/12.798138

[11] V. R. Weidner, J. J. Hsia, and B. Adams, "Laboratory intercomparison study of pressed polytetrafluoroethylene powder reflectance standards," Appl. Opt., vol. 24, no. 14, pp. 2225-2230, Jul 1985. [Online]. Available: http://ao.osa.org/abstract.cfm?URI=ao-24-14-2225

[12] V. R. Weidner and J. J. Hsia, "Reflection properties of pressed polytetrafluoroethylene powder," J. Opt. Soc. Am., vol. 71, no. 7, pp. 856-861, Jul 1981. [Online]. Available: http://www.osapublishing.org/abstract.cfm?URI=josa-71-7-856

[13] P. Kadkhoda, D. Ristau, and F. von Alvensleben, "Total scatter measurements in the DUV/VUV," vol. 3578, 1999, pp. 544-554. [Online]. Available: http://dx.doi.org/10.1117/12.344390 
[14] C. Silva, J. Pinto da Cunha, A. Pereira, V. Chepel, M. I. Lopes, V. Solovov, and F. Neves, "Reflectance of polytetrafluoroethylene for xenon scintillation light," Journal of Applied Physics, vol. 107, no. 6, 2010. [Online]. Available: http://scitation.aip.org/content/aip/journal/jap/107/6/10.1063/1.3318681

[15] S. Bricola, A. Menegolli, M. Prata, M. Prata, G. Raselli, M. Rossella, and C. Vignoli, "Noble-gas liquid detectors: measurement of light diffusion and reflectivity on commonly adopted inner surface materials," Nuclear Physics B - Proceedings Supplements, vol. 172, pp. 260 - 262, 2007. [Online]. Available: http://www.sciencedirect.com/science/article/pii/S0920563207006093

[16] C. Silva, "Reflection Distribution of the Fluoropolymers for the Xenon Scintillation Ligh," Ph.D. dissertation, University of Coimbra, Portugal, 2009.

[17] D. S. Akerib et al., "The Large Underground Xenon (LUX) Experiment," Nucl. Instrum. Meth., vol. A704, pp. 111-126, 2013.

[18] M. Yamashita, T. Doke, K. Kawasaki, J. Kikuchi, and S. Suzuki, "Scintillation response of liquid Xe surrounded by PTFE reflector for gamma rays," Nucl. Instrum. Meth., vol. A535, no. 3, pp. 692 - 698, 2004. [Online]. Available: http://www.sciencedirect.com/science/article/pii/S0168900204016638

[19] F. Neves, V. Chepel, V. Solovov, A. Pereira, M. I. Lopes, J. P. da Cunha, P. Mendes, A. Lindote, C. P. Silva, R. F. Marques, and A. J. P. L. Policarpo, "Performance of a chamber for studying the liquid xenon response to $\gamma$-rays and nuclear recoils," IEEE Transactions on Nuclear Science, vol. 52, no. 6, pp. 2793-2800, Dec 2005.

[20] F. Pedrotti and L. Pedrotti, Introduction to Optics. Prentice Hall, 1993.

[21] M. Miyajima, S. Sasaki, and E. Shibamura, "Absolute number of photons produced by alpha-particles in liquid and gaseous xenon," Nucl. Instrum. Meth., vol. B63, no. 3, pp. 297 - 308, 1992. [Online].

Available: http://www.sciencedirect.com/science/article/pii/0168583X92951136

[22] E. Aprile, R. Mukherjee, and M. Suzuki, "Measurements of the lifetime of conduction electrons in liquid xenon," Nucl. Instrum. Meth., vol. A300, no. 2, pp. 343 - 350, 1991. [Online]. Available: http://www.sciencedirect.com/science/article/pii/016890029190446W

[23] G. Bakale, U. Sowada, and W. F. Schmidt, J. Phys. Chem., vol. 80, p. 2556, 1976.

[24] A. Morozov, V. Solovov, R. Martins, F. Neves, V. Domingos, and V. Chepel, "ANTS2 package: simulation and experimental data processing for Anger camera type detectors," Journal of Instrumentation, vol. 11, no. 04, p. P04022, 2016. [Online]. Available: http://stacks.iop.org/1748-0221/11/i=04/a=P04022

[25] S. Agostinelli et al., "GEANT4 - a simulation toolkit," Nucl. Instrum. Meth., vol. A506, no. 3, pp. 250 303, 2003. [Online]. Available: http://www.sciencedirect.com/science/article/pii/S0168900203013688

[26] G. M. Seidel, R. E. Lanou, and W. Yao, "Rayleigh scattering in rare-gas liquids," Nucl. Instrum. Meth., vol. A489, pp. 189-194, 2002.

[27] V. N. Solovov, V. Chepel, M. I. Lopes, and A. Hitachi, "Measurement of the refractive index and attenuation length of liquid xenon for its scintillation light," Nucl. Instrum. Meth., vol. A516, pp. 462-474, 2004.

[28] B. Karlsson and C. G. Ribbing, "Optical constants and spectral selectivity of stainless steel and its oxides," Journal of Applied Physics, vol. 53, pp. 6340-6346, Sep. 1982.

[29] R. Gupta, J. H. Burnett, U. Griesmann, and M. Walhout, "Absolute refractive indices and thermal coefficients of fused silica and calcium fluoride near 193 nm," Appl. Opt., vol. 37, no. 25, pp. 5964-5968, Sep 1998. [Online]. Available: http://ao.osa.org/abstract.cfm?URI=ao-37-25-5964 
[30] M. Szydagis et al., "A Detailed Look at the First Results from the Large Underground Xenon (LUX) Dark Matter Experiment," in Proceedings, 10th International Symposium on Cosmology and Particle Astrophysics (CosPA 2013): Honolulu, Hawaii, USA, November 12-15, 2013, 2014. [Online]. Available: https://inspirehep.net/record/1281497/files/arXiv:1402.3731.pdf

[31] F. Nicodemus, J. Richmond, NBS., S. U. d'America. National Bureau of Standards, J. Hsia, I. Ginsberg, T. Limperis, and U. S. N. B. of Standards, Geometrical Considerations and Nomenclature for Reflectance, ser. NBS monograph. U.S. Government Printing Office, 1977. [Online]. Available: https://books.google.com/books?id=Pgr2ugAACAAJ

[32] J. Haefner et al., "Reflectance dependence of polytetrafluoroethylene on thickness for xenon scintillation light," submitted for publication. [Online]. Available: https://arxiv.org/abs/1608.01717 